PROCEEDINGS OF THE

AMERICAN MATHEMATICAL SOCIETY

Volume 127, Number 10, Pages 2975-2979

S 0002-9939(99)04904-7

Article electronically published on April 28, 1999

\title{
A DIXMIER-SCHAEFER-ZHANG THEOREM FOR OPERATOR ALGEBRAS
}

\author{
L. J. BUNCE, KAZUYUKI SAITÔ, AND J. D. MAITLAND WRIGHT \\ (Communicated by David R. Larson)
}

\begin{abstract}
Schaefer and Zhang have recently obtained an analogue, for sequentially order continuous functionals on $C(K)$, of a much earlier theorem of Dixmier. In this note it is shown that the Schaefer-Zhang Theorem has a natural generalisation to non-commutative $C^{*}$-algebras. These results are obtained as consequences of our main theorem which is concerned with affine functions on compact convex sets.
\end{abstract}

\section{INTRODUCTION}

In an interesting article in these Proceedings [7], Schaefer and Zhang showed that a Baire measure on a compact Hausdorff space $K$ is sequentially order continuous as a linear functional on $C(K)$, the commutative $C^{*}$-algebra of continuous functions on $K$, if and only if it vanishes on meagre Baire sets of $K$. This, they point out, parallels an earlier result of Dixmier [3].

Our goal is a generalisation of the Schaefer-Zhang Theorem to non-commutative $C^{*}$-algebras. It turns out to be natural and convenient to first work in the even more general setting of affine functions on compact convex spaces. This note is intended to be accessible to any functional analyst and has been written so as not to require a specialist knowledge of either convexity or $C^{*}$-algebras by the reader.

\section{PRELIMINARIES}

Throughout this note $\mathcal{A}$ is a unital $C^{*}$-algebra. Let $A$ be the self-adjoint part of $\mathcal{A}$. Let us recall the close connections between operator algebras, partially ordered spaces and compact convex sets (see [2] for a lucid and detailed account). In particular, $A$ is a real Banach space and has a natural partial ordering induced by the proper closed cone of elements of the form $z z^{*}$. The unit element of the algebra, $I$, acts as an order unit for the partial ordering and the order unit norm on $A$ coincides with the algebra norm. Let $S$ be the state space of $\mathcal{A}$. Then $A$ is isometrically and order isomorphic to $A(S)$, the space of all (real-valued) affine continuous functions on $S$ equipped with the pointwise partial ordering and the supremum norm.

Let $A(X)$ be the space of all (real valued) affine continuous functions on $X$, where $X$ is a compact convex subset of a Hausdorff locally convex vector space. Let $A(X)$ be equipped with the natural partial ordering and norm. Let $I$ denote

Received by the editors August 1, 1997 and, in revised form, January 6, 1998.

1991 Mathematics Subject Classification. Primary 46L05, 28A60. 
the function on $X$ with the constant value 1 . Thus $I$ is an order unit. Let $A^{b}(X)$ be the space of all bounded affine functions on $X$. Then $A^{b}(S)$ can be identified with the selfadjoint part of the von Neumann algebra, $\mathcal{A}^{* *}$, the second dual of $\mathcal{A}$.

Definition 1. The Baire envelope of $A(X)$, denoted by $A^{\infty}(X)$, is the intersection of all subspaces of $A^{b}(X)$ which contain $A(X)$ and which are closed under pointwise limits of bounded monotonic sequences, i.e. $A^{\infty}(X)$ is the smallest subspace of $A^{b}(X)$ such that $A(X)$ is a subspace of $A^{\infty}(X)$ and, whenever $\left(a_{n}\right)(n=1,2, \ldots)$ is a bounded monotonic sequence in $A^{\infty}(X)$ and $a(x)=\lim a_{n}(x)$ for each $x$ in $X$, then $a$ is in $A^{\infty}(X)$.

It follows from the results of Pedersen $[5,6]$ that $A^{\infty}(S)+i A^{\infty}(S)$ can be identified with a monotone sequentially closed $C^{*}$-subalgebra of $\mathcal{A}^{* *}$. Pedersen calls this the 'Borel envelope' of the $C^{*}$-algebra $\mathcal{A}$. We shall denote this by $\mathcal{A}^{\infty}$.

Let $\partial X$ be the set of extreme points of $X$ and let $\partial X$ be equipped with the relative topology induced by the topology of $X$. When $K$ is a compact Hausdorff space then the state space of $C(K)$ can be identified with $P(K)$, the space of all probability measures on $K$, and $\partial P(K)$ can be identified with $K$. In general $\partial X$ is not compact but, by a delicate result of Choquet (see page 49 in [1]), it is always a Baire space, i.e. the Baire Category Theorem holds for $\partial X$.

The restriction map $\left.f \rightarrow f\right|_{K}$ maps $A^{\infty}(P(K))$ onto $B^{\infty}(K)$, the bounded Baire measurable functions on $K$.

\section{SEQUENTIALLY ORDER CONTINUOUS FUNCTIONALS}

Let $\phi$ be any non-zero positive linear functional on $A(X)$. Then $\phi(I)^{-1} \phi$ is a state of $A(X)$, that is, there is a unique $x_{\phi}$ in $X$ such that, for each $a$ in $A(X)$,

$$
\phi(a)=\phi(I) a\left(x_{\phi}\right) .
$$

Then the (generalised) Baire measure corresponding to $\phi$ is denoted by $\phi^{\infty}$, and given by

$$
\phi^{\infty}(a)=\phi(I) a\left(x_{\phi}\right)
$$

for all $a$ in $A^{\infty}(X)$.

Definition 2. Let $\left(a_{n}\right)(n=1,2, \ldots)$ be a bounded monotone decreasing sequence in $A(X)$. This sequence has infimum 0 if each $a_{n} \geq 0$, and whenever $b$ is in $A(X)$ and $b \leq a_{n}$ for each $n$, then $b \leq 0$.

Definition 3. Let $\phi$ be a positive linear functional on $A(X)$. Then $\phi$ is said to be sequentially order continuous if, whenever $\left(a_{n}\right)(n=1,2, \ldots)$ is a monotone decreasing sequence in $A(X)$ with infimum 0 , then $\lim \phi\left(a_{n}\right)=0$.

We recall that a subset $N$ of a topological space $T$ is nowhere dense if the closure of $N$ has empty interior; and that a subset $M$ of $T$ is meagre if it is the union of a countable collection of nowhere dense sets.

Lemma 4. Let $K$ be a compact Hausdorff space and let $\mu$ be a Baire measure on $K$. The following are equivalent:

(i) $\mu(F)=0$ whenever $F$ is a closed, nowhere dense, $G_{\delta}$ set in $K$.

(ii) Let $u$ be a non-negative, upper semi-continuous Baire measurable function on $K$ such that $\{t \in K: u(t)>0\}$ is meagre. Then $\mu(u)=0$. 
Proof. When $F$ is a closed $G_{\delta}$ subset of $K$ then $\chi_{F}$ is an upper semi-continuous Baire measurable function. So (ii) implies (i).

Now let us assume (i) and let $u$ satisfy the hypotheses of (ii). Since $K$ is compact $u$ is bounded above by $M$, say. For any $\varepsilon>0$, let $F(\varepsilon)=u^{-1}[\varepsilon, M]$. Then $F(\varepsilon)$ is a closed Baire set and hence (see page 221 in [4]) is a closed $G_{\delta}$ set. Since $F(\varepsilon)$ is meagre its interior is empty. So $\mu(F(\varepsilon))=0$. Since $u \leq \varepsilon+M \chi_{F(\varepsilon)}$ it follows that $\mu(u) \leq \varepsilon \mu(K)$. Since this holds for all $\varepsilon>0$ we have $\mu(u)=0$.

Definition 5. An affine function $b$ in $A^{\infty}(X)$ is said to be meagre if the set $\{x \in$ $\partial X: b(x) \neq 0\}$ is a meagre subset of $\partial X$.

Definition 6. A Baire upper semi-continuous affine function on $X$ is a function $u$ on $X$ such that there exists a monotone decreasing sequence $\left(a_{n}\right)(n=1,2, \ldots)$ in $A(X)$ with $u(x)=\lim _{n} a_{n}(x)$ for each $x$ in $X$.

Lemma 7. Let $\left(a_{n}\right)(n=1,2, \ldots)$ be a monotone decreasing sequence of nonnegative functions in $A(X)$. Let $u(x)=\lim a_{n}(x)$ for each $x$ in $X$. Then $\left(a_{n}\right)$ $(n=1,2, \ldots)$ has infimum 0 in $A(X)$ if, and only if, $u$ is meagre.

Proof. This is a specialisation of Corollary 3 in [9].

Theorem 8. Let $\phi$ be a positive linear functional on $A(X)$. Then $\phi$ is sequentially order continuous on $A(X)$ if, and only if, whenever $u$ is a non-negative, meagre, Baire upper semi-continuous affine function on $X$ then $\phi^{\infty}(u)=0$.

Proof. Let $\left(a_{n}\right)(n=1,2, \ldots)$ be a monotone decreasing sequence of non-negative functions in $A(X)$ with infimum 0 . Let $u(x)=\lim a_{n}(x)$ for each $x$ in $X$. Then $u$ is a non-negative, Baire upper semi-continuous affine function on $X$ which is meagre. We have

$$
\phi^{\infty}(u)=u\left(x_{\phi}\right)=\lim a_{n}\left(x_{\phi}\right)=\lim \phi\left(a_{n}\right) .
$$

Suppose that $\phi^{\infty}$ vanishes on every non-negative, meagre, Baire upper semi-continuous affine function on $X$. Then, by $(\#), \lim \phi\left(a_{n}\right)=0$. Hence $\phi$ is sequentially order continuous.

Conversely, let $\phi$ be sequentially order continuous. Let $u$ be any non-negative, meagre, Baire upper semi-continuous affine function on $X$. Then there exists a monotone decreasing sequence of affine continuous functions on $X,\left(a_{n}\right)(n=$ $1,2, \ldots)$, which decreases pointwise to $u$. Moreover, since $u$ is meagre the sequence has infimum 0 in $A(X)$. Since, by hypothesis, $\phi$ is sequentially order continuous, $\lim \phi\left(a_{n}\right)=0$. So, by $(\#), \phi^{\infty}(u)=0$.

Corollary 9 (Schaefer-Zhang). Let $K$ be a compact Hausdorff space. Let $\phi$ be a positive linear functional on $C(K)$. Then $\phi$ is sequentially order continuous if, and only if, the corresponding Baire measure vanishes on all closed, nowhere dense $G_{\delta}$ subsets of $K$.

Proof. By the theorem, $\phi$ is sequentially order continuous if, and only if, $\phi^{\infty}(u)=0$ for each non-negative, meagre upper semi-continuous Baire function on $K$. By Lemma 4 the latter condition is equivalent to $\phi^{\infty}$ vanishing on all closed, nowhere dense $G_{\delta}$ subsets of $K$. 


\section{OPERATOR ALGEBRAS}

In this section we shall obtain a generalised Schaefer-Zhang Theorem for $C^{*}$ algebras which is closely related to the $C^{*}$-algebra's minimal $\sigma$-completion. As before $\mathcal{A}$ is a unital $C^{*}$-algebra, with 'Borel' envelope $\mathcal{A}^{\infty}$ whose self-adjoint part can be identified with $A^{\infty}(S)$. See the discussion following Definition 1 . The second dual of $\mathcal{A}, \mathcal{A}^{* *}$, is identified with the von Neumann envelope of $\mathcal{A}$ in the universal representation of $\mathcal{A}$; see [8]. The self-adjoint part of $\mathcal{A}^{* *}$ can be identified with $A^{b}(S)$ and $\mathcal{A}^{\infty}$ is the smallest subalgebra of $\mathcal{A}^{* *}$ which contains $\mathcal{A}$ and is such that each bounded, monotonic sequence of self-adjoint operators in it converges (strongly) to an operator in $\mathcal{A}^{\infty}$.

Definition 10. A (sequentially) meagre upper semi-continuous positive operator (abbreviated SMUSC operator) is an operator $u$ in $\mathcal{A}^{\infty}$ such that there exists a monotone decreasing sequence $\left(a_{n}\right)(n=1,2, \ldots)$ in $A$ which converges strongly to $u$ and $\left(a_{n}\right)(n=1,2, \ldots)$ has infimum zero.

When $b$ is an operator in the self-adjoint part of $\mathcal{A}^{* *}$, we shall abuse our notation by also using $b$ to denote the (bounded affine) function defined on $S$ by giving it the value $s(b)$ for each $s$ in $S$. It follows from Lemma 7 that $u$ is a SMUSC operator if, and only if, the corresponding function is non-negative, meagre, and Baire upper semi-continuous.

Minimal $\sigma$-completions of $C^{*}$-algebras were introduced in [10]. Let us recall that $\mathcal{L}(\mathcal{A})$ is the smallest $\sigma$-ideal of $\mathcal{A}^{\infty}$ which contains all SMUSC operators and that $\mathcal{A}^{\infty} / \mathcal{L}(\mathcal{A})$ can be identified with the minimal $\sigma$-completion of $\mathcal{A}$.

Lemma 11. Let $u$ be a SMUSC operator in $\mathcal{A}^{\infty}$ and let $w$ be any invertible element of $\mathcal{A}$. Then $w^{*} u w$ is also a SMUSC operator in $\mathcal{A}$.

Proof. Let $\left(a_{n}\right)(n=1,2, \ldots)$ be a monotone decreasing sequence in $A$ converging to $u$. Since $u$ is a SMUSC operator, $\left(a_{n}\right)(n=1,2, \ldots)$ has 0 as its infimum.

Let $b \in A$. Now $b$ is a lower bound for $\left(w^{*} a_{n} w\right)(n=1,2, \ldots)$ if, and only if, $\left(w^{-1}\right)^{*} b w^{-1}$ is a lower bound for $\left(a_{n}\right)(n=1,2, \ldots)$. So $\left(w^{*} a_{n} w\right)(n=1,2, \ldots)$ has 0 for its infimum. Hence $w^{*} u w$ is a SMUSC operator.

For $\phi$ a state of $\mathcal{A}$, let $\phi^{\prime \prime}$ be the extension of $\phi$ to a normal state of $\mathcal{A}^{* *}$ and let $\phi^{\infty}$ be the restriction of $\phi^{\prime \prime}$ to $\mathcal{A}^{\infty}$. This is consistent with our earlier notation. Let $\left(\pi_{\phi}, H_{\phi}, \xi_{\phi}\right)$ be the GNS representation of $\mathcal{A}$ associated with $\phi$. Then the homomorphism $\pi_{\phi}: \mathcal{A} \rightarrow L\left(H_{\phi}\right)$ has a $\sigma$-normal extension to $\pi_{\phi}^{\infty}: \mathcal{A}^{\infty} \rightarrow L\left(H_{\phi}\right)$.

Theorem 12. Let $\phi$ be a state of a unital $C^{*}$-algebra $\mathcal{A}$. Then the following statements are equivalent.

(i) $\phi$ is sequentially order continuous.

(ii) The kernel of $\pi_{\phi}^{\infty}$ contains the $\sigma$-ideal $\mathcal{L}(\mathcal{A})$ of $\mathcal{A}^{\infty}$.

(iii) There exists a unique $\sigma$-normal state $\phi^{\wedge}$ on $\mathcal{A}^{\infty} / \mathcal{L}(\mathcal{A})$, the minimal $\sigma$ completion of $\mathcal{A}$, such that, for every $x$ in $\mathcal{A}^{\infty}$,

$$
\phi^{\infty}(x)=\phi^{\wedge}(x+\mathcal{L}(\mathcal{A})) .
$$

(iv) There exists a unique $\sigma$-normal state $\phi^{\wedge}$ on the minimal $\sigma$-completion of $\mathcal{A}$ which extends $\phi$.

Proof. Let us assume (i). Then $\phi^{\infty}(u)$ vanishes whenever $u$ is a SMUSC operator in $\mathcal{A}^{\infty}$. Since $\pi_{\phi} \mathcal{A} \xi_{\phi}$ is dense in $H_{\phi}$, to show that $\pi_{\phi}^{\infty}(u)$ is zero it suffices to show that 
it vanishes on $\pi_{\phi} w \xi_{\phi}$ for each $w$ in $\mathcal{A}$. The square of the norm of $\pi_{\phi}^{\infty}\left(u^{1 / 2}\right)\left(\pi_{\phi} w \xi_{\phi}\right)$ is $\phi^{\infty}\left(w^{*} u w\right)$.

Let $w$ be an invertible element of $\mathcal{A}$. By Lemma $11, w^{*} u w$ is a SMUSC operator and so, by (i), $\phi^{\infty}\left(w^{*} u w\right)=0$.

Now suppose that $w$ is any element of $\mathcal{A}$. It suffices to consider the situation where $w$ has norm less than 1 . Then $\left(w+i^{k}\right)$ is invertible for $k=2$ and 4 . So $\phi^{\infty}\left(\left(w+i^{k}\right)^{*} u\left(w+i^{k}\right)\right)$ vanishes for $k=2$ and 4 . On adding we get

$$
2 \phi^{\infty}\left(w^{*} u w\right)+2 \phi^{\infty}(u)=0
$$

It follows that each SMUSC operator is in the kernel of $\pi_{\phi}^{\infty}$. Since this kernel is a $\sigma$-ideal and since $\mathcal{L}(\mathcal{A})$ is the smallest $\sigma$-ideal of $\mathcal{A}^{\infty}$ which contains the SMUSC operators [10] we have established (ii). (iv).

It now follows that (ii) implies (iii) and, on restricting $\phi^{\infty}$ to $\mathcal{A}$, that (iii) implies

Let us assume (iv) and let $\left(a_{n}\right)(n=1,2, \ldots)$ be a monotone decreasing sequence in $A$ with infimum 0 . Then this sequence has the same infimum in the minimal $\sigma$-completion of $\mathcal{A}$. So $\phi^{\wedge}\left(a_{n}\right)$ converges to zero, that is, $\phi\left(a_{n}\right)$ converges to zero. So $\phi$ is a sequentially order continuous functional on $\mathcal{A}$.

\section{REFERENCES}

1. E. M. Alfsen, Compact convex sets and boundary integrals, Springer 1971. MR 56:3615

2. L. Asimov and A. J. Ellis, Convexity theory and its applications in functional analysis, LMS Monograph 16, Academic Press, 1980. MR 82m:46009

3. J. Dixmier, 'Sur certains espaces considérés par M. H. Stone', Summa Brasil. Math 2 (1951), 151-182. MR 14:69e

4. P. R. Halmos, Measure theory, Springer, 1974.

5. G. K. Pedersen, 'On weak and monotone $\sigma$-closures of $C^{*}$-algebras', Commun. Math. Phys. 11 (1969), 221-226. MR 39:1987

6.,$C^{*}$-algebras and their automorphism groups, LMS Monographs 14, Academic Press, 1979. MR 81e: 46037

7. H. H. Schaefer and X-D. Zhang, 'Dixmier's theorem for sequentially order continuous Baire measures on compact spaces', Proc. Amer. Math. Soc. 125 (1997), 93-99. MR 97c:28030

8. M. Takesaki, Theory of operator algebras I, Springer 1979. MR 81e:46038

9. J. D. M. Wright, 'On approximating concave functions by convex functions', Bull. London Math. Soc. 5 (1973), 221-222. MR 48:12058

10. ' 'On minimal $\sigma$-completions of $C^{*}$-algebras', Bull. London Math. Soc. 6 (1974), 168174. MR 51:6437

Mathematical Institute, Tohoku University, Sendai 980, Japan

E-mail address: saito@math.tohoku.ac.jp

Analysis and Combinatorics Research Centre, Mathematics Department, University of Reading, Reading RG6 6AX, England

E-mail address: j.d.m.wright@rdg.ac.uk 